This article was downloaded by: [National Taiwan University]

On: 31 August 2009

Access details: Access Details: [subscription number 731897496]

Publisher Taylor \& Francis

Informa Ltd Registered in England and Wales Registered Number: 1072954 Registered office: Mortimer House, 37-41 Mortimer Street, London W1T 3JH, UK

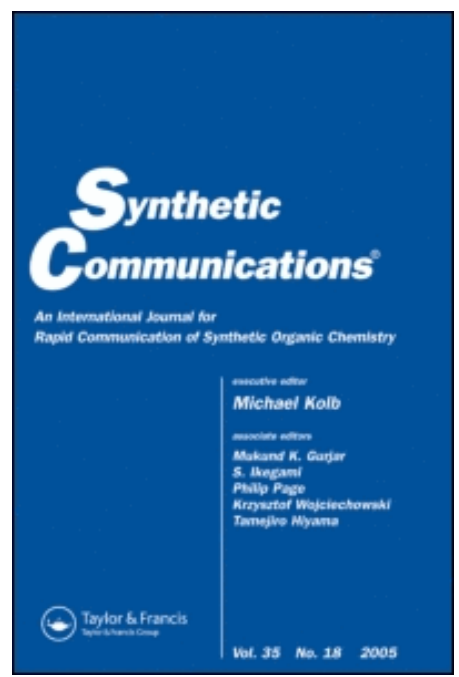

\title{
Synthetic Communications
}

Publication details, including instructions for authors and subscription information:

http://www.informaworld.com/smpp/title content=t713597304

\section{Preparation of Functionalized Imidazolium Salts under Microwave Irradiation}

Shih-Kang Fu a; Shiuh-Tzung Liu a

a Department of Chemistry, National Taiwan University, Taipei, Taiwan

Online Publication Date: 01 June 2006

To cite this Article Fu, Shih-Kang and Liu, Shiuh-Tzung(2006)'Preparation of Functionalized Imidazolium Salts under Microwave Irradiation',Synthetic Communications,36:14,2059 - 2067

To link to this Article: DOI: $10.1080 / 00397910600634464$

URL: http://dx.doi.org/10.1080/00397910600634464

\section{PLEASE SCROLL DOWN FOR ARTICLE}

Full terms and conditions of use: http://www.informaworld.com/terms-and-conditions-of-access.pdf

This article may be used for research, teaching and private study purposes. Any substantial or systematic reproduction, re-distribution, re-selling, loan or sub-licensing, systematic supply or distribution in any form to anyone is expressly forbidden.

The publisher does not give any warranty express or implied or make any representation that the contents will be complete or accurate or up to date. The accuracy of any instructions, formulae and drug doses should be independently verified with primary sources. The publisher shall not be liable for any loss, actions, claims, proceedings, demand or costs or damages whatsoever or howsoever caused arising directly or indirectly in connection with or arising out of the use of this material. 


\title{
Preparation of Functionalized Imidazolium Salts under Microwave Irradiation
}

\author{
Shih-Kang Fu and Shiuh-Tzung Liu \\ Department of Chemistry, National Taiwan University, Taipei, Taiwan
}

\begin{abstract}
A direct alkylation of a substituted imidazole to prepare the corresponding functionalized ionic liquid has been developed in excellent yields under microwave irradiation.
\end{abstract}

Keywords: Imidazolium, ionic liquid, microwave irridiation

From an environmental perspective, ionic liquids provide an alternative reaction medium for chemical reactions. ${ }^{[1]}$ Among various ionic liquids, simple alkyl substituted 1,3-dialkyl-imidazolium cations associated with weakly coordinating anions are the most popular used in biphasic catalysis because of their distinct physical-chemical properties. ${ }^{[1,2]}$ More recently, developments of the presence of appropriate functional groups as part of ionic liquid molecules to increase the immobilization of catalysts or facilitate the separation process represent a new area of interest. ${ }^{[3]}$ Thus, a few of this type of ionic liquids, such as imidazolium-based ones with amine, ${ }^{[4]}$ amido, ${ }^{[5]}$ hydroxy, ${ }^{[6]}$ carboxylate,${ }^{[7]}$ urea, ${ }^{[8]}$ perfluoro-chains, ${ }^{[9]}$ and nitriles, ${ }^{[3]}$ are known.

Conventional heating in refluxing solvents is a typical way to prepare 1,3dialkylimidazolium halides. Compared to the time-consuming processes of conventional methods, a microwave-assisted chemical reaction has been applied to synthesize ionic liquids. ${ }^{[10,11]}$ In the past two decades, the use of microwave ovens in chemical synthesis and analysis has grown because of the advantages in reducing reaction times, improving yield, and simplifying procedures. The preparation of functionalized ionic liquids using a solventfree approach has not reported in literature. ${ }^{[4-9]}$

Received in Japan March 7, 2006

Address correspondence to Shiuh-Tzung Liu, Department of Chemistry, National Taiwan University, Taipei 106, Taiwan. E-mail: stliu@ntu.edu.tw 


\section{RESULTS AND DISCUSSION}

The microwave-assisted reaction was conducted in a round-bottom flask using a Discovery microwave heating apparatus with a thermal controller (CEM Corp., Matthews, NC). This apparatus allows the reaction flask to be equipped with condenser fitted into a microwave-irradiation chamber (see the experimental section). Typically, a mixture of equal molar amounts $(6.5 \mathrm{mmol})$ of 1 -methylimidazole and $\gamma$-bromobutyonitrile in a 20 -mL flask was irradiated at $180 \mathrm{~W}$ at $180^{\circ} \mathrm{C}$ for $30 \mathrm{~s}$. Upon completion of the reaction, the pale yellow, viscous oil was washed with dichloromethane $(20 \mathrm{~mL} \times 2)$ to remove the unreacted starting materials. The residue was dried under vacuum overnight, and the obtained material was analytical pure.

All reactions studied in this work are shown in Scheme 1. As a starting point, we studied the microwave-assisted coupling of 1-methylimidazole with 3-triethoxysilylpropyl chloride. Entries 1-5 of Table 1 show the results of this optimization. It appeared that the period of irradiation and reaction temperature were critical for the reaction. The best conditions for the preparation of $\mathbf{1}$ is irradiation at microwave power of $245 \mathrm{~W}$ for $40 \mathrm{~s}$. Either a higher or a lower power of irradiation in this reaction gave decomposition or poorer yield. The scale for the reaction is irrelevant to the irradiation period employed. Because of the moisture sensitivity of the triethoxy silyl group, the reaction had to be carried out under a dry nitrogen atmosphere; otherwise the condensation leading to $\mathrm{Si}-\mathrm{O}-\mathrm{Si}$ took place, which caused a decrease in the yield of $\mathbf{1}$. However, it can be carried out under aerobic

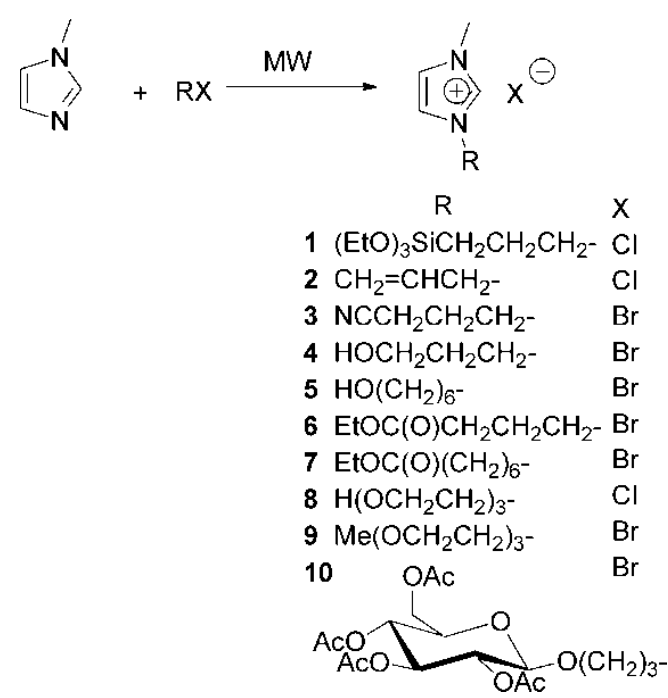

Scheme 1. Preparation of functionalized 1,3-disubstituted imidazolium-based ionic liquids. 
Table 1. Results for the reactions of 1-methylimidazole with various alkyl halides ${ }^{\mathrm{a}}$

\begin{tabular}{|c|c|c|c|c|c|c|c|c|c|}
\hline Entry & $\begin{array}{l}\text { Quantity of } \\
\text { reactant (mmol) }\end{array}$ & Product & $\begin{array}{l}\text { Power of } \\
\text { MW (W) }\end{array}$ & $\mathrm{T}(\mathrm{s})$ & $\begin{array}{l}\text { Temp. } \\
\left({ }^{\circ} \mathrm{C}\right)\end{array}$ & Atm. & Yield $^{b}$ & Physical property & Ref. \\
\hline 1 & 6.5 & 1 & 245 & 40 & 245 & $\mathrm{~N}_{2}$ & $95 \%$ & Pale yellow liquid & {$[12]$} \\
\hline 3 & 6.5 & 1 & 245 & 25 & 140 & $\mathrm{~N}_{2}$ & trace & - & \\
\hline 4 & 6.5 & 1 & 185 & 40 & 145 & $\mathrm{~N}_{2}$ & trace & - & \\
\hline 5 & 6.5 & 1 & 245 & 40 & 185 & $\mathrm{~N}_{2}$ & $93 \%$ & Pale yellow liquid & \\
\hline 8 & 6.5 & 4 & 100 & 25 & 175 & air & $92 \%$ & Lemon yellow liquid & [14] \\
\hline 9 & 6.5 & 5 & 100 & 35 & 180 & air & $90 \%$ & Colorless liquid & [15] \\
\hline 10 & 3.4 & 6 & 100 & 35 & 168 & air & $90 \%$ & Pale yellow liquid & [16] \\
\hline 11 & 4.6 & 7 & 180 & 30 & 180 & air & $81 \%$ & Lemon yellow liquid & \\
\hline 12 & 5.0 & 8 & 250 & 90 & 185 & air & $90 \%$ & Colorless liquid & \\
\hline 13 & 2.9 & 9 & 250 & 90 & 185 & air & $85 \%$ & Colorless liquid & \\
\hline
\end{tabular}

${ }^{a}$ Reaction conditions: an equal molar amount of 1-methylimidazole and RX in flask without using any solvent.

${ }^{b}$ Isolated yield and characterized by ${ }^{1} \mathrm{H}$ NMR. 
conditions for other substrates. All products were characterized by ${ }^{1} \mathrm{H}$ NMR and elemental analysis. The optimum reaction conditions for reactions of 1-methylimidazole with various alkyl halides are summarized in Table 1.

As shown in Table 1, various alkyl halides were able to react with imidazole to give the corresponding ionic liquid in excellent yields (entries $6-13$ ). For example, the production of $\mathbf{1 0}$ was achieved by adopting $30 \mathrm{~W}$ at $110^{\circ} \mathrm{C}$, whereas the compound 8 was with $250 \mathrm{~W}$ at $185^{\circ} \mathrm{C}$. For the acetylated glucose derivative, the reaction provided a moderate yield by using $30 \mathrm{~W}$ of microwave at $110^{\circ} \mathrm{C}$. Attempts to improve the yield by varying the microwave powers and temperatures failed, indicating an intrinsic property of this substrate.

All functionalized imidazolium halides except $\mathbf{1 0}$ are in liquid phase at room temperature. Compound $\mathbf{1 0}$ is in a solid form, which becomes liquid at temperatures greater than $90-93^{\circ} \mathrm{C}$. As for solubility, those containing $-\mathrm{OH}$ of polyether groups are water- and methanol-soluble materials. In fact, compound $\mathbf{8}$ is completely miscible with water. Of course, the halide anions of this series of ionic liquids also increase their solubility toward water. Similarly, the alkylation of 1-propyl-2-methylimidazole proceeded smoothly to give the corresponding ionic liquid in excellent yield (Scheme 2). The reaction conditions employed are comparable to the preparation of the related 1,3-disubstituted imidazolium-based ionic liquids (Table 2).

Reaction of 1-bromo-3-chloropropane with 1-methylimidazole under microwave irradiation yielded a mixture of $\mathbf{1 4}, \mathbf{1 5},{ }^{[17]}$ and $\mathbf{1 6}^{[18]}$ (Scheme 3). It shows that the poor selectivity of nucleophilic substitution of imidazole toward alkyl bromide versus chloride under microwave-assisted conditions even with a lower power irradiation. Even under mild conditions with lower power $(30 \mathrm{~W})$, this reaction still provided a mixture of products. However, the reaction proceeded poorly at the temperature lower than $100^{\circ} \mathrm{C}$. All these compounds were not able to be obtained in pure form because of the anion.

3-Aminopropyl chloride was also tested as a substrate for the alkylation. A mixture of an equalmolar amount of 3-aminopropyl chloride hydrochloride

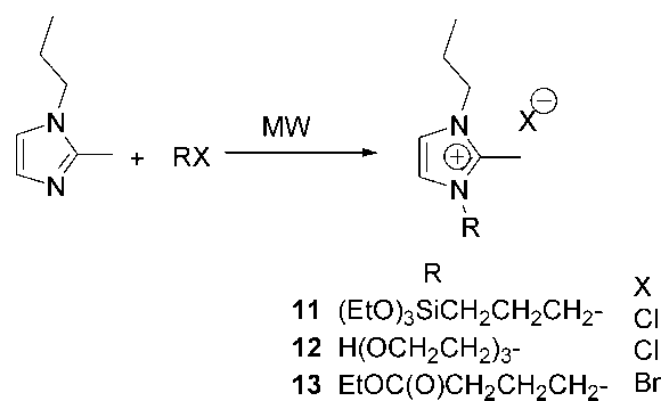

Scheme 2. Preparation of functionalized 1,2,3-trisubstituted imidazolium-based ionic liquids. 
Table 2. Results for preparation of 1,2,3-trisubstituted imidazolium-based ionic liquids

\begin{tabular}{lccccc}
\hline Product & Power $(\mathrm{W})$ & $\mathrm{T}(\mathrm{s})$ & Temp. $\left({ }^{\circ} \mathrm{C}\right)$ & Yield $(\%)$ & Physical property \\
\hline $\mathbf{1 1}$ & 235 & 40 & 185 & 75 & Pale yellow liquid \\
$\mathbf{1 2}$ & 180 & 50 & 180 & 89 & Pale yellow liquid \\
$\mathbf{1 3}$ & 105 & 30 & 180 & 85 & Pale yellow liquid \\
\hline
\end{tabular}

salt, 1-methylimidazole, and triethylamine was irradiated at $170^{\circ} \mathrm{C}$ with the power setting of $180 \mathrm{~W}$ for $90 \mathrm{~s}$ to provide the desired ionic liquid $\mathbf{1 7}$ in $65 \%$ yield accompanied by the formation of azetidine Eq. (1). This outcome shows that the alkylation takes place with imidazole nitrogen superior to the amine site. However, the use of excess 3-aminopropyl chloride salt can provide $\mathbf{1 7}$ in a high yield.
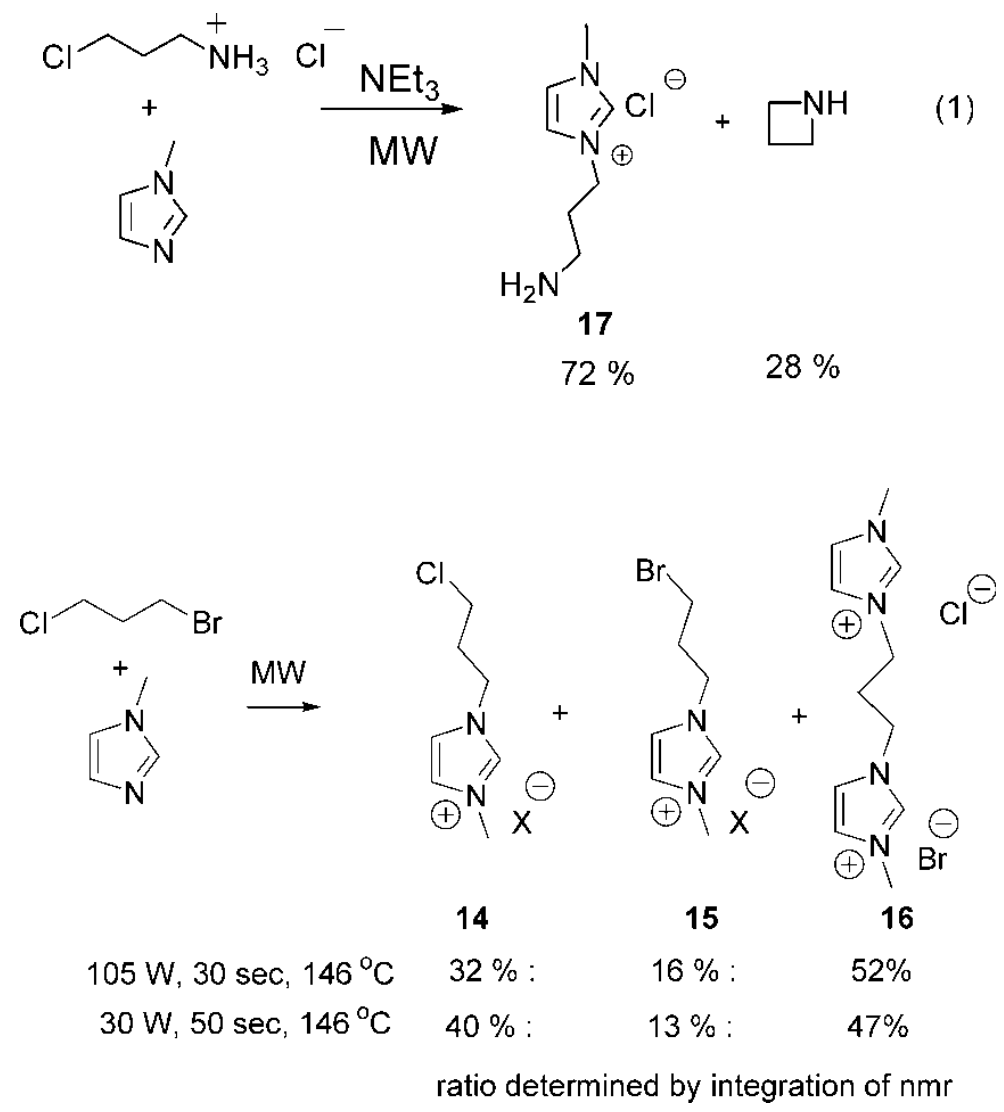

Scheme 3. Reaction of imidazole with 3-chloro-1-bromopropane. 
In summary, we have demonstrated a much faster and more efficient method for the preparation of functionalized imidazolium-based ionic liquid in high yields. Work on these functionalized ionic liquids continues and is expected to illustrate interesting new results.

\section{EXPERIMENTAL}

Nuclear magnetic resonance spectra were recorded in $\mathrm{CDCl}_{3}$ or acetone- $\mathrm{d}_{6}$ on either a Bruker AM-300 or Avance 400 spectrometer. Chemical shifts are given in parts per million relative to $\mathrm{Me}_{4} \mathrm{~S}$ for ${ }^{1} \mathrm{H}$ and ${ }^{13} \mathrm{C}$ NMR. Infrared spectra were measured on a Nicolet Magna-IR 550 spectrometer (series-II) as $\mathrm{KBr}$ pallets, unless otherwise noted. Microwave irradiation was carried out in a Discovery microwave heating apparatus with temperature controller (CEM Corp., Matthews, NC). The reaction temperature reported was based on this readout. Chemicals and solvents were of analytical grade and used as received unless otherwise stated. $\alpha$-Bromotetraacetyl-L-glucose was prepared from the earlier reported procedures. ${ }^{[19]}$

\section{General Procedure}

An equal molar amount of 1-methylimidazole and alkyl halides in a 20-mL flask was set up as shown in Fig. 1 and was irradiated at a certain level of power with programming the temperature for a certain period of reaction time. Upon completion of the reaction, the pale yellow, viscous oil was washed with dichloromethane $(20 \mathrm{~mL} \times 2)$ to remove the unreacted starting materials and was dried under vacuum overnight. Spectroscopic data of the obtained compounds 1-6 are essentially identical to the literature reported. ${ }^{[3,12-16]}$

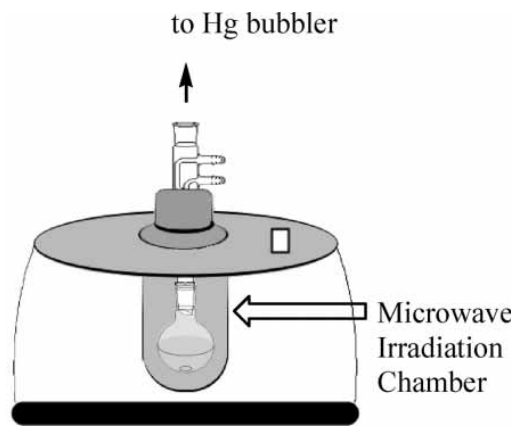

Figure 1. Microwave-assisted heating apparatus. 


\section{Data}

Compound 7. $v_{\max } \mathrm{KBr} / \mathrm{cm}^{-1} 3164,2952,2866,1726\left(v_{\mathrm{C}=\mathrm{O}}\right), 1653\left(v_{\mathrm{C}=\mathrm{C}}\right)$, 1573; ${ }^{1} \mathrm{H}$ NMR $\left(300 \mathrm{MHz}, \mathrm{CDCl}_{3}\right): \delta 9.99(\mathrm{~s}, 1 \mathrm{H}), 7.53(\mathrm{~s}, 1 \mathrm{H}), 7.47(\mathrm{~s}, 1 \mathrm{H})$, $4.20(\mathrm{t}, 2 \mathrm{H}, J=7.31 \mathrm{~Hz}), 3.93(\mathrm{~s}, 3 \mathrm{H}), 3.91(\mathrm{q}, 2 \mathrm{H}, J=7.05 \mathrm{~Hz}), 2.16$ $(\mathrm{t}, J=7.29 \mathrm{~Hz}), 1.80(\mathrm{~m}, 2 \mathrm{H}), 1.50(\mathrm{~m}, 2 \mathrm{H}), 1.24(\mathrm{~m}, 2 \mathrm{H}), 1.07(\mathrm{t}, 3 \mathrm{H}$, $J=7.18 \mathrm{~Hz}$ ). MS found $[\mathrm{M}-\mathrm{Br}]^{+} 239$ for $\mathrm{C}_{13} \mathrm{H}_{23} \mathrm{~N}_{2} \mathrm{O}_{2}$; anal. calcd. for $\mathrm{C}_{13} \mathrm{H}_{23} \mathrm{BrN}_{2} \mathrm{O}_{2}$ : C, 48.91; $\mathrm{H}, 7.26 ; \mathrm{N}, 8.78$. Found for $\mathrm{C}, 45.45 ; \mathrm{H}, 7.15$; N, 9.46.

Compound 8. $v_{\max } \mathrm{KBr} / \mathrm{cm}^{-1} 3552\left(v_{\mathrm{O}-\mathrm{H}}\right), 3164,2872,1653\left(v_{\mathrm{C}=\mathrm{C}}\right), 1587$, 1116; ${ }^{1} \mathrm{H}$ NMR $\left(300 \mathrm{MHz}, \mathrm{D}_{2} \mathrm{O}\right): \delta 8.75(\mathrm{~s}, 1 \mathrm{H}), 7.52(\mathrm{~s}, 1 \mathrm{H}), 7.45(\mathrm{~s}, 1 \mathrm{H})$, $4.39(\mathrm{t}, 2 \mathrm{H}, J=4.6 \mathrm{~Hz}), 3.91(\mathrm{~s}, 3 \mathrm{H}), 3.89(\mathrm{~m}, 2 \mathrm{H}), 3.72(\mathrm{~m}, 6 \mathrm{H}), 3.60$ $(\mathrm{m}, 2 \mathrm{H})$. MS found $[\mathrm{M}-\mathrm{Cl}]^{+} 215$ for $\mathrm{C}_{10} \mathrm{H}_{19} \mathrm{~N}_{2} \mathrm{O}_{3}$; anal. calcd. for $\mathrm{C}_{10} \mathrm{H}_{19} \mathrm{ClN}_{2} \mathrm{O}_{3}$ : C, 47.9; H, 7.64; N, 11.19. Found: C, 47.21; H, 7.83; N, 11.37.

Compound 9. $v_{\max } \mathrm{KBr} / \mathrm{cm}^{-1} 3164,2873,1646,1566,1103 ;{ }^{1} \mathrm{HNMR}$ $\left(300 \mathrm{MHz}, \mathrm{CDCl}_{3}\right): \delta 9.81(\mathrm{~s}, 1 \mathrm{H}), 7.66(\mathrm{~s}, 1 \mathrm{H}), 7.47(\mathrm{~s}, 1 \mathrm{H}), 3.97(\mathrm{~s}, 3 \mathrm{H})$, $3.79(\mathrm{t}, 2 \mathrm{H}, J=4.58 \mathrm{~Hz}), 3.52(\mathrm{~m}, 8 \mathrm{H}), 3.25(\mathrm{~s}, 3 \mathrm{H})$. MS found [M-Br] ${ }^{+}$ 229 for $\mathrm{C}_{11} \mathrm{H}_{21} \mathrm{~N}_{2} \mathrm{O}_{3}$; anal. calcd. for $\mathrm{C}_{11} \mathrm{H}_{21} \mathrm{BrN}_{2} \mathrm{O}_{3}$ : C, 42.73; $\mathrm{H}, 6.85 ; \mathrm{N}$, 9.06. Found: C, 42.59; H, 7.19; N, 8.98.

Compound 10. Mp: 90-93 ${ }^{\circ}$; $v_{\max } \mathrm{KBr} / \mathrm{cm}^{-1} 3171,2939,2879,1726$ $\left(v_{\mathrm{C}=\mathrm{O}}\right), 1653,1587,1089 ;{ }^{1} \mathrm{H} \mathrm{NMR}\left(300 \mathrm{MHz}, \mathrm{CDCl}_{3}\right): \delta 10.05(\mathrm{~s}, 1 \mathrm{H})$, $7.38(\mathrm{~s}, 1 \mathrm{H}), 7.31(\mathrm{~s}, 1 \mathrm{H}), 5.15(\mathrm{t}, 1 \mathrm{H}, J=9.6 \mathrm{~Hz}), 5.02(\mathrm{t}, 1 \mathrm{H}, J=9.9 \mathrm{~Hz})$, $4.85(\mathrm{t}, 1 \mathrm{H}, J=8.0 \mathrm{~Hz}), 4.50(\mathrm{~d}, 1 \mathrm{H}, J=8.0 \mathrm{~Hz}), 4.39(\mathrm{~m}, 2 \mathrm{H}), 4.20$ $(\mathrm{m}, 1 \mathrm{H}), 4.06(\mathrm{~s}, 3 \mathrm{H}), 3.71(\mathrm{~m}, 2 \mathrm{H}), 3.62(\mathrm{~m}, 2 \mathrm{H}), 2.20(\mathrm{~m}, 2 \mathrm{H}), 2.05$ $(\mathrm{s}, 1 \mathrm{H}), 2.02(\mathrm{~s}, 1 \mathrm{H}), 2.00(\mathrm{~s}, 1 \mathrm{H}), 1.95(\mathrm{~s}, 1 \mathrm{H})$. MS found $[\mathrm{M}-\mathrm{Br}]^{+} 472$ for $\mathrm{C}_{20} \mathrm{H}_{29} \mathrm{~N}_{2} \mathrm{O}_{10}$; anal. calcd. for $\mathrm{C}_{20} \mathrm{H}_{29} \mathrm{BrN}_{2} \mathrm{O}_{10}$ : C, 44.70; H, 5.44; N, 5.21. Found: C, 44.26; H, 5.45; N, 4.99.

Compound 11. $v_{\max } \mathrm{KBr} / \mathrm{cm}^{-1} 3144,2980,2933,2849,1640,1586,1076$; ${ }^{1} \mathrm{HNMR}\left(300 \mathrm{MHz}, \mathrm{CDCl}_{3}\right): \delta 7.66(\mathrm{~s}, 1 \mathrm{H}), 7.51(\mathrm{~s}, 1 \mathrm{H}), 4.27(\mathrm{t}, 2 \mathrm{H}$, $J=7.3 \mathrm{~Hz}), 4.08(\mathrm{t}, 6 \mathrm{H}, J=7.3 \mathrm{~Hz}), 3.92(\mathrm{q}, 2 \mathrm{H}, J=7.2 \mathrm{~Hz}), 2.65(\mathrm{~s}, 3 \mathrm{H})$, $2.34(\mathrm{t}, 2 \mathrm{H}, J=7.2 \mathrm{~Hz}), 1.97(\mathrm{~m}, 2 \mathrm{H}), 1.72(\mathrm{~m}, 2 \mathrm{H}), 0.82(\mathrm{t}, 9 \mathrm{H}$, $J=7.3 \mathrm{~Hz}$ ). MS found $[\mathrm{M}-\mathrm{Cl}]^{+} 287$ for $\mathrm{C}_{13} \mathrm{H}_{27} \mathrm{~N}_{2} \mathrm{O}_{3} \mathrm{Si}$; anal. calcd. for $\mathrm{C}_{13} \mathrm{H}_{27} \mathrm{ClN}_{2} \mathrm{O}_{3} \mathrm{Si}: \mathrm{C}, 48.35 ; \mathrm{H}, 8.43 ; \mathrm{N}, 8.68$. Found: $\mathrm{C}, 47.21 ; \mathrm{H}, 7.83$; $\mathrm{N}, 11.37$.

Compound 12. $v_{\max } \mathrm{KBr} / \mathrm{cm}^{-1} 3403\left(v_{\mathrm{O}-\mathrm{H}}\right), 3144,2952,2886,1653,1593$, 1129; ${ }^{1} \mathrm{H}$ NMR $\left(300 \mathrm{MHz}, \mathrm{CDCl}_{3}\right): \delta 7.81(\mathrm{~s}, 1 \mathrm{H}), 7.49(\mathrm{~s}, 1 \mathrm{H}), 4.43(\mathrm{t}, 2 \mathrm{H}$, $J=4.5 \mathrm{~Hz}), 4.10(\mathrm{t}, 2 \mathrm{H}, J=7.2 \mathrm{~Hz}), 3.81 \quad(\mathrm{t}, 2 \mathrm{H}, J=4.5 \mathrm{~Hz}), 3.52$ $(\mathrm{m}, 8 \mathrm{H}), 2.65(\mathrm{~s}, 3 \mathrm{H}), 1.75(\mathrm{~m}, 2 \mathrm{H}), 0.88(\mathrm{t}, 3 \mathrm{H}, J=5.2 \mathrm{~Hz})$. MS found $[\mathrm{M}-\mathrm{Cl}]^{+} 257$ for $\mathrm{C}_{13} \mathrm{H}_{25} \mathrm{ClN}_{2} \mathrm{O}_{3}$; anal. calcd. for $\mathrm{C}_{13} \mathrm{H}_{25} \mathrm{ClN}_{2} \mathrm{O}_{3}$ : C, 53.33; H, 8.61; N, 9.57. Found: C, 53.18; H, 8.98; N, 9.44. 
Compound 13. $v_{\max } \mathrm{KBr} / \mathrm{cm}^{-1}$ 3139, 2974, 2883, 1727, 1640, 1587; ${ }^{1} \mathrm{HNMR}\left(300 \mathrm{MHz}, \mathrm{CDCl}_{3}\right): \delta 7.65(\mathrm{~s}, 1 \mathrm{H}), 7.54(\mathrm{~s}, 1 \mathrm{H}), 4.19(\mathrm{t}, 2 \mathrm{H}$, $J=11.2 \mathrm{~Hz}), 3.43(\mathrm{~s}, 9 \mathrm{H}), 2.72(\mathrm{~s}, 3 \mathrm{H}), 1.79(\mathrm{~m}, 2 \mathrm{H}), 0.84(\mathrm{t}, 3 \mathrm{H}$, $J=18.4 \mathrm{~Hz}), 0.54(\mathrm{t}, 3 \mathrm{H}, \quad J=12.6 \mathrm{~Hz}) . \mathrm{MS}$ found $[\mathrm{M}-\mathrm{Br}]^{+} 239$ for $\mathrm{C}_{13} \mathrm{H}_{23} \mathrm{BrN}_{2} \mathrm{O}_{2}$; anal. calcd. for $\mathrm{C}_{13} \mathrm{H}_{23} \mathrm{BrN}_{2} \mathrm{O}_{2}$ : C, 48.91; H, 7.26; N, 8.78. Found for C, 48.96; H, 7.93; N, 8.96.

Compound 17. $v_{\max } \mathrm{KBr} / \mathrm{cm}^{-1} 3455\left(v_{\mathrm{N}-\mathrm{H}}\right), 3157,2963,2753,1634,1579$; ${ }^{1} \mathrm{HNMR}\left(300 \mathrm{MHz}, \mathrm{D}_{2} \mathrm{O}\right): \delta 8.65(\mathrm{~s}, 1 \mathrm{H}), 7.39(\mathrm{~s}, 1 \mathrm{H}), 7.32(\mathrm{~s}, 1 \mathrm{H}), 4.19$ $(\mathrm{t}, 2 \mathrm{H}, J=7.3 \mathrm{~Hz}), 3.75(\mathrm{~s}, 3 \mathrm{H}), 2.93(\mathrm{~m}, 2 \mathrm{H}), 2.16(\mathrm{~m}, 2 \mathrm{H})$. MS found $[\mathrm{M}-\mathrm{Cl}]^{+} 140$ for $\mathrm{C}_{7} \mathrm{H}_{14} \mathrm{~N}_{3}$; anal. calcd. for $\mathrm{C}_{7} \mathrm{H}_{14} \mathrm{ClN}_{3}$ : C, 47.86; H, 8.03. Found for C, 47.66; H, 7.83.

\section{ACKNOWLEDGMENT}

This research was supported by the National Science Council (NSC93-2113M002-004), Taiwan, China.

\section{REFERENCES}

1. Recentreviews: (a) Wilkes, J. S. Properties of ionic liquid solvents for catalysis. J. Mol. Catal. A, Chem 2004, 214, 11-17; (b) Dzyuba, S. V.; Bartsch, R. A. Recent advances in applications of room-temperature ionic liquid/supercritical $\mathrm{CO}_{2}$ systems. Angew. Chem. Int. Ed. 2003, 42, 148-150; (c) Dupont, J.; de Souza, R. F.; Suarez, P. A. Z. Ionic liquid (molten salt) phase organometallic catalysis. Chem. Rev. 2002, 102, 3667-3692; (d) Handy, S. T. Greener solvents: Room temperature ionic liquids from biorenewable sources. Chem.-Eur. J. 2003, 9, 2938-2944.

2. Suarez, P. A. Z.; Dullius, J. E. L.; Einloft, S.; de Souza, R. F.; Dupont, J. Synthesis and physical-chemical properties of ionic liquids based on 1-n-butyl-3-methylimidazolium cation. J. Chim. Phys. 1998, 95, 1626-1639.

3. Zhao, D.; Fei, Z.; Scopelliti, R.; Dyson, P. J. Synthesis and characterization of ionic liquids incorporating the nitrile functionality. Inorg. Chem. 2004, 43, 2197-2205

4. Herrmann, W. A.; Köcher, C.; Goossen, L. J.; Artus, G. R. J. Heterocyclic carbenes: A high-yielding synthesis of novel, functionalized $\mathrm{N}$-heterocyclic carbenes in liquid ammonia. Chem.-Eur. J. 1996, 2, 1627-1636.

5. Lee, K.-M.; Lee, Y.-T.; Lin, I. J. B. Supramolecular liquid crystals of amide functionalized imidazolium salts. J. Mater. Chem. 2003, 13, 1079-1084.

6. Branco, L. C.; Rosa, J. N.; Moura Ramos, J. J.; Alfons, C. A. M. Preparation and characterization of new room temperature ionic liquids. Chem.-Eur. J. 2002, 8, 3671-3677.

7. (a) Cole, A. C.; Jensen, J. L.; Ntai, I.; Tran, K. L. T.; Weaver, K. J.; Forbes, D. C.; Davis, J. H., Jr. Novel Bronsted acidic ionic liquids and their use as dual solventcatalysts. J. Am. Chem. Soc. 2002, 124, 5962-5963; (b) Holbrey, J. D.; Reichert, W. M.; Tkatchenko, I.; Bouajila, E.; Walter, O.; Tommasi, I.; 
Roger, R. D. 1,3-Dimethylimidazolium-2-carboxylate: The unexpected synthesis of an ionic liquid precursor and carbene- $\mathrm{CO}_{2}$ adduct. Chem. Commun. 2003, $28-29$.

8. Visser, A. E.; Swatloski, R. P.; Reichert, W. M.; Mayton, R.; Sheff, S.; Wierzbicki, A.; Davis, J. H., Jr.; Rogers, R. D. Task-specific ionic liquids for the extraction of metal ions from aqueous solutions. Chem. Commun. 2001, $135-136$.

9. Merrigan, T. L.; Bates, E. D.; Dorman, S. C.; Davis, J. H., Jr. New fluorous ionic liquids function as surfactants in conventional room-temperature ionic liquids. Chem. Commun. 2000, 2051-2502.

10. (a) Varma, R. S.; Namboodiri, V. V. Solvent-free preparation of ionic liquids using a household microwave oven. Pure Appl. Chem. 2001, 73, 1309-1313; (b) Namboodiri, V. V.; Varma, R. S. Microwave-assisted preparation of dialkylimidazolium tetrachloroaluminates and their use as catalysts in the solvent-free tetrahydropyranylation of alcohols and phenols. Chem. Commun. 2002, 342-343.

11. (a) Thanh, G. V.; Pegot, B.; Loupy, A. Solvent-free microwave-assisted preparation of chiral ionic liquids from (-)-N-methylephedrine. Eur. J. Org. Chem. 2004, 1112-1116; (b) Ranu, B. C.; Jana, R.; Dey, S. S. An efficient and green synthesis of 2-arylbenzothiazoles in an ionic liquid, [pmIm] Br under microwave irradiation. Chem. Lett. 2004, 33, 274-275; (c) Peng, Y.; Song, G. Microwaveassisted clean synthesis of 6-aryl-2,4-diamino-1,3,5-triazines in [bmim] $\left[\mathrm{PF}_{6}\right]$. Tetrahedron Lett. 2004, 45, 5313-5316.

12. Chi, Y. S.; Lee, J. K.; Lee, S.-G.; Choi, I. S. Control of wettability by anion exchange on $\mathrm{Si} / \mathrm{SiO}_{2}$ surfaces. Langmuir 2004, 20, 3024-3027.

13. Ricciardi, F.; Romanchick, W. A.; Joullie, M. M. 1,3-Dialkylimidazolium salts as latent catalysts in the curing epoxy-resins. J. Polym. Sci., Polym. Lett. 1983, 21, 633-638.

14. Walker, A. J.; Bruce, N. C. Combined biological and chemical catalysis in the preparation of oxycodone. Tetrahedron 2004, 60, 561-568.

15. Dubreuil, J. F.; Bazureau, J. P. Rate accelerations of 1,3-dipolar cycloaddition reactions in ionic liquids. Tetrahedron Lett. 2000, 7351-7355.

16. Horvath, A. Hofmann-type elimination in the efficient $N$-alkylation of azolesimidazole and benzimidazole. Synthesis 1994, 102-106.

17. Zhang, D.; Chen, J.; Liang, Y.; Zhou, H. Facile synthesis of novel ionic liquids containing dithiocarbamate. Synth. Commun. 2005, 35, 521-526.

18. Poyatos, M.; Mas-Marza, E.; Sanau, M.; Peris, E. Synthesis and reactivity of new chelate- $N$-heterocyclic biscarbene complexes of ruthenium. Inorg. Chem. 2004, 43, 1793-1798.

19. Potter, A. L.; Sowden, J. C.; Hassid, W.; Doudoroff, M. $\alpha$-L-Glucose-1-phosphate. J. Am. Chem. Soc. 1948, 70, 1751-1752. 